\title{
Associação entre comportamentos externalizantes e baixo desempenho escolar: uma revisão de estudos prospectivos e longitudinais
}

\author{
Lylla Cysne Frota D'Abreu \\ Universidade de Potsdam, Alemanha \\ Edna Maria Marturano \\ Faculdade de Medicina da Universidade de São Paulo - Ribeirão Preto
}

\begin{abstract}
Resumo
Foi feito um levantamento na literatura de estudos prospectivos e longitudinais investigando a associação entre problemas de comportamento externalizantes e baixo desempenho escolar no ensino fundamental, no período de 1990 a 2006. Por meio dos sistemas PsycInfo, Medline, Lilacs, Scielo e Web of Science, foram selecionados e adquiridos 18 artigos, classificados em quatro categorias: estudos com amostras clínicas; estudos comparando grupos com ou sem comorbidade; estudos que buscam testar modelos de trajetória de desenvolvimento; estudos sobre precursores da associação entre problemas de comportamento e baixo desempenho escolar. A análise dos artigos evidencia que a co-ocorrência de baixo desempenho escolar e problemas externalizantes sugere a influência de variáveis antecedentes, como condições adversas na família e baixo nível socioeconômico. Indica, ainda, que a associação traz mau prognóstico às crianças, como comorbidades com transtornos psiquiátricos, posteriores problemas acadêmicos e de comportamento anti-social, evidenciando a situação de risco psicossocial em que se encontram.
\end{abstract}

Palavras-chave: baixo desempenho escolar; problemas de comportamento externalizantes; TDAH; estudo longitudinal.

\begin{abstract}
Association between externalizing behavior and underachievement: a review of prospective and longitudinal studies. A survey in the literature of prospective and longitudinal studies was made investigating the association between externalizing behavior and underachievement in the period of 1990-2006. It was used PsycInfo, Medline, Lilacs, Scielo and Web of Science systems and it was selected and acquired 18 articles, classified in four categories: studies with clinical samples; studies comparing groups with or without comorbidity; studies that try to establish or to test models of developmental trajectory; studies of precursors of the association between behavior problems and underachievement. The analysis of articles evidences that the co-occurrence of underachievement and externalizing behavior suggests the influence of antecedent variables, such as adverse conditions in the family and low socio-economic level. It indicates that the association brings bad prognostic to the children, such as comorbidity with psychiatric disorders, later academic problems, and antisocial behavior, evidencing the psychosocial risk situation in that they are.
\end{abstract}

Keywords: underachievement; externalizing behavior; ADHD; longitudinal study.

A presença expressiva de crianças com queixa escolar na demanda por atendimento psicológico (Barbosa \& Silvares, 1994) pode estar relacionada à co-ocorrência de baixo desempenho escolar com outros problemas adaptativos (Hinshaw, 1992). Baixo desempenho escolar é caracterizado como desempenho, em notas ou tarefas, abaixo de um nível esperado para a idade, habilidade e potencial de um indivíduo. É, portanto, uma discrepância entre a aptidão acadêmica e seu desempenho (Ellis \& Bernard, 2006). Sete em cada dez crianças encaminhadas à rede de saúde com queixa de baixo desempenho escolar apresentam sintomas emocionais e/ou comportamentais em nível clínico (Marturano, Toller, \& Elias, 2005), uma taxa muito acima daquelas indicadas em estudos recentes de prevalência na população brasileira, entre 10 e $20 \%$ (Cury \& Golfeto, 2003).

Comportamentos marcados por oposição, agressão, hiperatividade, impulsividade, desfio e manifestações antisociais são classificados como externalizantes, em oposição a padrões de comportamento internalizantes - disforia, retraimento, medo e ansiedade (Achenbach \& Edelbrock, 1978). A associação entre comportamentos externalizantes e habilidades acadêmicas pobres está bem estabelecida na literatura (Dodge \& 
Pettit, 2003) e há relato de identificação precoce, já na pré-escola (Arnold, 1997). Estudos têm investigado como essa associação influencia a trajetória de desenvolvimento das pessoas afetadas. Masten et al. (2005), em seguimento de 20 anos, constataram que problemas externalizantes evidentes na meninice prejudicam o desempenho na adolescência e o desempenho rebaixado, por sua vez, está associado a problemas internalizantes no início da idade adulta.

Hinshaw (1992) realizou extensa revisão bibliográfica investigando a relação entre baixo desempenho escolar e problemas de comportamento externalizantes. Na seção do artigo dedicada a estudos longitudinais, com um tempo de seguimento de no mínimo um ano, o autor refere treze artigos focalizando crianças que cursavam o ensino fundamental no início da investigação. Todos trabalharam com amostras da comunidade. Apesar da diversidade de métodos, algumas tendências foram identificadas.

A primeira diz respeito a variáveis antecedentes. Há indícios de influência de fatores familiares, dificuldades de fala e de linguagem e imaturidade neurodesenvolvimental, como substrato da associação, porém os resultados são inconclusivos, devido a problemas metodológicos nas investigações (Hinshaw, 1992). A segunda refere-se a aspectos desenvolvimentais dos problemas externalizantes associados ao desempenho escolar pobre. Os estudos mostraram, em geral, que, na infância, desatenção e hiperatividade têm maior associação com desempenho acadêmico que agressividade, no entanto, na adolescência, comportamento anti-social e delinqüência estão claramente ligados a problemas acadêmicos (Hinshaw, 1992).

Hinshaw apontou, em sua revisão, questões relevantes suscitadas pela análise dos estudos longitudinais. No que se refere à etiologia da associação, há dificuldade para estabelecer relações de causa e efeito. Dada a heterogeneidade de ambos os domínios - problema de comportamento externalizante e fracasso escolar - e a provável complexidade da associação entre ambos, modelos causais lineares, ou mesmo um modelo bidirecional, podem ser considerados simplistas. Modelos multifatoriais, que consideram fatores intra-individuais (temperamento, problemas de fala), familiares (nível sócio-econômico, tamanho da família) e escolares, parecem mais pertinentes para explicar a associação (Hinshaw, 1992).

Outra questão apontada pelo autor foi que, apesar de a literatura identificar de forma substancial os efeitos adversos de problemas de comportamento externalizantes e baixo desempenho escolar, ainda pouco se sabia, à época, sobre os riscos psicossociais e os fatores de proteção associados à persistência e à remissão do problema na adolescência (Hinshaw, 1992). Os estudos longitudinais apresentam, então, fundamental importância para a compreensão da co-ocorrência, porque são potencialmente mais informativos, mais passíveis a inferências causais complexas, avaliam as conseqüências adversas na adolescência e no período adulto, e possibilitam identificar fatores de risco e proteção associados a diferentes trajetórias de desenvolvimento (Hinshaw, 1992).

A revisão feita por Hinshaw parece ter tido grande impacto na literatura. Da década de noventa até os dias atuais, novos estudos longitudinais foram realizados, com o propósito de investigar relações preditivas, controlar adequadamente variáveis intervenientes e discutir questões conceituais. Considerando que a produção de conhecimento nesse campo pode contribuir para práticas de saúde mental junto à população infantil, o presente estudo se orienta em direção a essas pesquisas. Seu objetivo foi identificar, na literatura científica indexada, estudos prospectivos e longitudinais, investigando a associação entre problemas de comportamento externalizantes e baixo desempenho escolar no ensino fundamental, publicados depois daquela revisão, no período de 1990 a 2006.

\section{Método}

O levantamento foi feito por pesquisa nas bases de dados PsycInfo, Medline, LILACS, SciELO e Web of Science, e por busca manual mediante consulta às referências bibliográficas dos artigos obtidos nas bases de dados. Para a busca eletrônica, utilizaram-se as seguintes combinações de palavras-chave: 1. (externalizing AND school underachievement AND child AND elementary grades) AND (longitudinal OR prospective OR follow-up); 2. (underachievement AND behavior AND child) AND (longitudinal OR prospective OR follow-up) e 3. (impulsivity OR hyperactivity OR distractibility) AND underachievement AND (longitudinal OR prospective OR follow-up). Nas bases SciELO e LILACS foi feita uma busca adicional com as palavras-chave (longitudinal AND criança), (prospectivo AND criança), (seguimento AND criança).

Os critérios de inclusão foram: relatos de pesquisa empírica, de língua portuguesa, inglesa ou castelhana; participantes com idade abaixo de 13 anos na primeira coleta de dados; pesquisas com pelo menos duas fases de coleta de dados, sendo a segunda realizada pelo menos 12 meses após a primeira. Os critérios de exclusão foram: participantes com enfermidade física ou necessidades especiais (deficiência mental, sensorial ou física); pesquisas que avaliavam propostas de intervenção; estudos com populações específicas (negros, indígenas, migrantes, crianças adotadas); estudos genéticos.

\section{Resultados e Discussão}

Aplicados os critérios de inclusão e exclusão foram selecionados 18 artigos, sendo 11 nos indexadores internacionais e sete por busca manual nos artigos localizados. Os estudos foram classificados em quatro categorias: estudos com amostras clínicas; estudos comparando grupos com ou sem comorbidade; estudos que buscam estabelecer ou testar modelos de trajetória de desenvolvimento; estudos sobre precursores da associação entre problemas de comportamento e baixo desempenho escolar.

Um sumário dos artigos nas quatro categorias é apresentado a seguir.

Estudos com amostras clínicas. Três artigos focalizavam amostras de crianças com hiperatividade provenientes de clínicas psiquiátricas ou psicológicas (Barkley, Fischer, Edelbrock, \& Smallish, 1990; Biederman et al., 1996; Fischer, Barkley, Fletcher, \& Smallish, 1993). Este conjunto de estudos prioriza a investigação do curso do transtorno e não a trajetória de 
desenvolvimento da criança.

Biederman et al. (1996) investigaram, em crianças com TDAH, a presença de comorbidade com outros transtornos psiquiátricos e a presença de prejuízos cognitivos, acadêmicos e adaptativos. As variáveis investigadas em um ano e quatro anos de seguimento foram desempenho escolar, transtornos psiquiátricos, funcionamento cognitivo e social e nível sócioeconômico. Os resultados mostraram que o grupo com TDAH apresentava no seguimento QI mais baixo, mais dificuldades em leitura e aritmética, maior desajustamento social, mais conflitos em família e taxas significativamente mais altas de comorbidade com outros transtornos psiquiátricos (conduta, humor, ansiedade, tiques e linguagem). A prevalência destas comorbidades tendia a ficar mais alta à medida que a criança se desenvolvia.

Os estudos de Barkley et al. (1990) e Fischer et al. (1993) utilizaram a mesma amostra para a realização de análises diferentes. Barkley et al. (1990) investigaram os problemas psiquiátricos de um grupo de crianças hiperativas, comparado a um grupo controle. As variáveis analisadas no seguimento de oito anos foram nível sócio-econômico, dados demográficos, mudanças de domicílio e de emprego dos pais, desempenho acadêmico, história de saúde mental, social e médica do adolescente, saúde mental dos pais e comportamento anti-social do pai. Os resultados mostraram que crianças com diagnóstico estabelecido de hiperatividade permaneciam com os sintomas por até oito anos. Na adolescência, mais de $80 \%$ continuavam a ter o problema; $60 \%$ apresentavam transtorno oposicional e/ ou de conduta; e apresentavam resultados acadêmicos e sociais mais negativos, maior número de atos anti-sociais, mais uso de cigarro e maconha, situação familiar menos estável (mudança de residência, de emprego do cuidador) e maior risco de transtorno familiar, quando comparadas ao grupo de adolescentes sem diagnóstico prévio de hiperatividade.

Fischer et al. (1993) investigaram as variáveis preditoras de problemas psiquiátricos, sociais, acadêmicos e de ajustamento emocional na adolescência, em indivíduos diagnosticados com hiperatividade oito anos antes. Na avaliação inicial foram analisadas complicações no parto, problemas médicos e desenvolvimentais, temperamento na pré-escola, impulsividadehiperatividade, problemas de conduta, ansiedade e características familiares (nível de educação materna, atos anti-sociais paternos e adversidade). No seguimento de oito anos, foram avaliados desempenho acadêmico, hiperatividade, sintomas de comportamento desafiador e de transtorno de conduta, problemas emocionais, competência social e ajustamento materno. Os resultados mostraram que a competência acadêmica e cognitiva na infância predizia habilidades acadêmicas na adolescência; impulsividade-hiperatividade na infância e atos anti-sociais paternos estavam associados a posterior comportamento oposicional-desafiador; e apenas desobediência na infância, e não hiperatividade, predizia problemas de encarceramento.

Estes estudos fornecem indícios de que crianças impulsivashiperativas estão em risco para futuras conseqüências negativas no seu ajustamento. A hiperatividade, no entanto, não pode ser considerada como um único preditor destes problemas posteriores de ajustamento, mas sim, a sua associação com outras desvantagens psicossociais, o que sugere tratar-se de uma condição de vulnerabilidade para comportamento oposicionaldesafiador.

Estudos comparando grupos com ou sem associação entre dificuldades de leitura e problemas de comportamento externalizante. Os artigos classificados nesta categoria trabalharam com amostras não clínicas, recrutadas na comunidade, e buscaram investigar as conseqüências adversas da associação entre dificuldades de leitura e problemas externalizantes: Chadwick, Taylor, Taylor, Heptinstall e Danckaerts (1999) e Pisecco, Baker, Silva e Brooke (1996) tiveram como ponto de partida a associação entre dificuldades de leitura e hiperatividade; Smart, Sanson e Prior (1996) analisaram a associação entre dificuldades de leitura e problemas de comportamento.

Em todos os estudos, quatro grupos foram formados: a. grupo apenas com hiperatividade/problemas de comportamento; b. grupo apenas com dificuldades de leitura; c. grupo com hiperatividade/problemas de comportamento e dificuldades de leitura; d. grupo controle.

Pisecco et al. (1996) identificaram crianças com TDAH e com idade inicial de 5 anos e as acompanharam até os 18 anos. As variáveis analisadas segundo pais e professores foram: habilidade de leitura, problemas de comportamento, sintomas de desatenção e hiperatividade e transtorno de conduta. Os resultados mostraram que, em casa, os grupos $\mathrm{H}$ e $\mathrm{DL}+\mathrm{H}^{1}$ desenvolveram mais comportamentos hiperativos, enquanto que, na escola, os grupos DL, H, DL $+\mathrm{H}$ apresentaram mais comportamentos hiperativos e anti-sociais que o grupo controle. Isto sugere que há determinados problemas que surgem na escola, mas não em casa, possivelmente porque estes grupos têm na escola diferentes demandas. Dentre todos, o grupo DL $+\mathrm{H}$ apresentou significativamente mais comportamentos anti-sociais que qualquer outro grupo.

Chadwick et al. (1999) e Smart et al. (1996) utilizaram a escala comportamental de Rutter para pais e professores para identificar problemas de hiperatividade ou de comportamento, com uma idade inicial de 7-8 anos. Smart et al. (1996) testaram hipóteses causais e temporais acerca da associação entre problemas de comportamento e dificuldades de leitura, com seguimento de dois anos. As variáveis que analisaram foram desempenho em leitura e problemas de comportamento segundo relato de pais e professores na avaliação inicial; e habilidade de leitura, ortografia, inteligência, problemas de comportamento (segundo relato de pais e professores) e nível sócio econômico no seguimento. Os resultados mostraram que o grupo com comorbidade obteve os piores resultados, apresentando, no seguimento, mais problemas de ansiedade-medo e hostilidadeagressividade no relato dos pais e professores, e progresso significativamente mais pobre em leitura quando comparado ao grupo DL, sugerindo que problemas de comportamento podem exacerbar dificuldades de leitura. O grupo DL se manteve estável no decorrer do tempo. Sua identificação aos 7-8 anos se mostrou confiável e sua recuperação espontânea, improvável. Diferenças de gênero também foram identificadas na associação entre dificuldades de leitura e problemas de comportamento, dois anos depois. Dois terços dos meninos identificados inicialmente 
apenas com dificuldade em leitura apresentaram problemas de comportamento no seguimento, enquanto dois terços das meninas com dificuldades de leitura não apresentaram problemas de comportamento, sugerindo que o caminho da associação é específico para o gênero.

Chadwick et al.(1999) investigaram a associação entre hiperatividade e baixo desempenho escolar em meninos, com seguimento de nove anos. Na avaliação inicial, foram avaliados problemas emocionais e de comportamento, desempenho em leitura, QI, complicações perinatais, neurológicas e de comunicação e adversidades sócio-econômicas. No seguimento, foram avaliados problemas emocionais e de comportamento, desempenho em leitura e QI. Os resultados mostraram que o grupo $\mathrm{H}+\mathrm{DL}$ apresentava mais problemas de comportamento anti-social e desafiador nas medidas de linhas de base e no follow-up, escores mais baixos em exames escolares e índices mais altos de evasão escolar, maior probabilidade de terem recebido terapia fonoaudiológica e maior evidência de adversidades sócio-econômicas. $\mathrm{O}$ grupo $\mathrm{H}+\mathrm{DL}$ e o grupo DL apresentaram mais atraso na compreensão e precisão na leitura que o grupo controle. Não houve diferenças significativas quanto ao QI. Este estudo não encontrou indícios de que hiperatividade exacerba dificuldades de leitura.

Os resultados dos estudos apontam que a comorbidade $\mathrm{DL}+\mathrm{H}$ prediz trajetória mais desfavorável, com dificuldades emocionais e de conduta, que DL e $\mathrm{H}$ isoladamente. Os resultados de Chadwick et al.(1999) e Pisecco et al. (1996) são convergentes ao mostrar que o grupo com comorbidade apresenta na adolescência mais comportamentos opositores e anti-sociais que os demais grupos. Chadwick et al.(1999), diferente de Smart et al. (1996), não encontraram indícios de que $\mathrm{H}$ exacerba DL. O tempo de seguimento variou entre os dois estudos, e o transtorno de hiperatividade pode assumir formas diferentes ao longo do tempo, o que pode explicar essa aparente inconsistência nos resultados. Uma hipótese plausível para conciliar os resultados dos dois estudos seria que a presença de $\mathrm{H}$ interfere no desenvolvimento da leitura apenas na fase de aquisição desta habilidade, perdendo importância à medida que estratégias de decifração do código escrito, efetivas ou não, são automatizadas, demandando menos investimento de atenção por parte do leitor.

Verifica-se, também, no estudo de Pisecco et al. (1996), a importância do relato tanto de pais como de professores quanto ao comportamento da criança, que pode apresentar problemas de comportamento "gerais" ou "situacionais", como por exemplo, problemas de comportamento somente na escola.

Estudos que buscam estabelecer ou testar modelos de trajetórias de desenvolvimento. Enquadram-se neste grupo sete artigos que têm interesse em investigar a trajetória percorrida por crianças que apresentam problemas externalizantes associados ao baixo desempenho acadêmico. Todos trabalharam com amostras não clínicas, recrutadas na comunidade, identificando os casos de interesse por meio de procedimentos de triagem e/ou diagnóstico.

Quatro artigos discutem o modelo de trajetória proposto por Fergusson, Horwood e Lynskey (1993) e apresentam resultados que confirmam esse modelo (Fergusson et al., 1993; Fergusson,
Lynskey, \& Horwood, 1997; McGee, Prior, Williams, Smart, \& Sanson, 2002; Rapport, Scanlan, \& Denney, 1999); um artigo discute a relação entre o desempenho em leitura e a delinqüência juvenil (Williams \& McGee, 1994); um artigo investiga o curso da relação entre TDAH e a competência acadêmica, incluindo fatores familiares, como transtorno psiquiátrico materno (Latimer et al. 2003); e um artigo analisa as características da trajetória da criança com baixo desempenho acadêmico e sua relação com pais e professores (McCall, Scott, \& Lau, 2000).

O modelo construído por Fergusson et al. (1993) apresenta três proposições: 1. Problemas de atenção são variáveis correlacionadas com problemas de conduta; 2 . Dificuldades de atenção influenciam diretamente o desempenho acadêmico, mas não o comportamento anti-social posterior; 3. Problemas de conduta influenciam posterior comportamento anti-social, mas não influenciam o desempenho acadêmico. As maiores implicações deste modelo são: 1. Problemas de atenção, na ausência de problemas de conduta, estão associados a maior risco de fracasso escolar, mas não aumentam o risco de comportamento anti-social; 2. Problemas de atenção em combinação com problemas de conduta estão associados tanto ao fracasso escolar quanto ao comportamento anti-social.

Fergusson et al. (1993) procuraram avaliar a relação entre transtorno de conduta e déficit de atenção na meninice intermediária e comportamento delinqüente e desempenho acadêmico na adolescência. As variáveis investigadas foram problemas de comportamento (aos seis, oito e dez anos), desempenho acadêmico (aos treze anos) e comportamento delinqüente precoce (aos doze e treze anos). Inteligência, posição social, escolaridade e qualidade da relação parental, assim como gênero, foram incluídas na análise como variáveis "confundidoras". Seus resultados mostraram que transtorno de conduta, na ausência de déficit de atenção, está associado a tendências futuras à delinqüência, mas não ao baixo desempenho acadêmico. E déficit de atenção, na ausência de desordem de conduta, está associado ao baixo desempenho acadêmico, mas não a comportamento delinqüente.

Fergusson et al. (1997) investigaram a associação entre dificuldades de atenção e conseqüências psicossociais e cognitivas negativas. Foram avaliadas, aos oito anos, dificuldades de atenção, e aos dezoito, desempenho escolar, comportamento ofensivo e abuso de substâncias. Os resultados mostraram que problemas de atenção levam ao baixo desempenho escolar, e que problemas de atenção associados a problemas de conduta resultam em baixo desempenho escolar e comportamento antisocial, confirmando, portanto, o modelo proposto pelos autores.

McGee et al. (2002) examinaram as conseqüências adversas, comportamentais e acadêmicas, de crianças com hiperatividade; compararam os resultados com os de crianças com dificuldades de leitura. A hiperatividade, o comportamento anti-social e o desempenho em leitura, no início do estudo (cinco a oito anos), foram examinados como preditores de conseqüências adversas em desempenho acadêmico e comportamento, no seguimento de dez anos, incluindo-se no modelo, variáveis da história familiar, como isolamento, abuso de substâncias e problemas no relacionamento parental. Os resultados mostraram uma forte associação entre hiperatividade na infância e dificuldades 
escolares, problemas de atenção e leitura empobrecida na adolescência e uma associação entre dificuldades de leitura na infância e problemas de leitura e evasão escolar na escola secundária. No entanto, dificuldade de atenção não foi precursora de comportamento anti-social no seguimento.

Rapport et al. (1999) tiveram como objetivos replicar o modelo proposto por Fergusson et al. (1993) e investigar a associação entre déficit de atenção e desempenho acadêmico, mediante a formulação de um modelo de trajetória dual, que considera aspectos comportamentais e cognitivos. Baseados em pesquisas anteriores, os autores hipotetizaram que esta associação poderia ser mediada pela performance na sala de aula (hipótese comportamental) e por vigilância (hipótese cognitiva). As variáveis analisadas, na avaliação inicial, foram inteligência, desempenho acadêmico, comportamento disruptivo, medidas cognitivas (vigilância, memória) e nível sócio-econômico; no seguimento de três e quatro anos, avaliou-se o desempenho acadêmico. Os resultados corroboraram os achados de Fergusson et al. (1993) e apontaram que o modelo de trajetória dual é consistente: comportamento na sala de aula e habilidades cognitivas servem como importantes mediadores entre déficit de atenção, QI e posterior desempenho acadêmico. Segundo este modelo, problemas de conduta estão relacionados a baixo desempenho acadêmico quando associados a TDAH e QI; TDAH está relacionado a desempenho acadêmico por duas trajetórias, uma comportamental e outra cognitiva; e na trajetória cognitiva, vigilância representa uma habilidade essencial para a memória.

Latimer et al. (2003) procuraram investigar a trajetória de crianças com TDAH em relação ao desempenho acadêmico e ajustamento social no fim da adolescência, e identificar qual o papel que fatores sócio-econômicos, familiares e da própria criança poderiam ter neste percurso. Estes autores analisaram, na primeira avaliação (sete a onze anos), características sociodemográficas, fatores familiares (ajustamento materno, coesão familiar, práticas parentais habilidosas de controle, comunicação e envolvimento e psicopatologia dos pais), hiperatividade, aptidão intelectual e desempenho acadêmico. No seguimento de quatro anos, avaliaram fatores familiares (transtornos psiquiátricos), desempenho acadêmico, ajustamento emocional e comportamental. No seguimento de seis anos, focalizaram desempenho acadêmico, ajustamento comportamental, uso de medicação psicotrópica, de serviços de saúde mental e de escola especial. Segundo seus resultados, crianças com TDAH identificado no início da avaliação apresentavam pior desempenho acadêmico e ajustamento comportamental seis anos depois, na adolescência. A associação foi mediada pela presença de uma pobre regulação emocional e comportamental durante a infância intermediária. Além disso, ajustamento materno, habilidades dos pais e bom desempenho acadêmico protegiam a criança com TDAH contra adversidades.

Williams e McGee (1994) procuraram examinar as relações entre dificuldades de leitura e comportamento anti-social na infância e leitura e comportamento delinqüente na adolescência, e avaliar o quanto essa trajetória era influenciada por desvantagens familiares (baixo nível sócio-econômico, família monoparental, separação dos pais, depressão materna, tamanho da família e baixo suporte social). As variáveis investigadas no estudo foram desempenho em leitura (dos sete aos quinze anos), problemas de comportamento (aos sete e aos nove anos), comportamento antisocial (aos sete anos), comportamento delinqüente e problemas de conduta (aos quinze anos), e desvantagens familiares (aos sete, aos nove e aos quinze anos). Os autores observaram que comportamento anti-social na infância prediz fortemente delinqüência aos quinze anos, especialmente em meninos, além de causar prejuízo à leitura por reduzir envolvimento com as tarefas acadêmicas. Por outro lado, não foi encontrada relação direta entre leitura e comportamento delinqüente posterior.

O estudo de McCall et al. (2000) difere dos demais artigos por ter como ponto de partida apenas o baixo desempenho escolar, e não problemas de atenção, hiperatividade e conduta. Seus objetivos eram caracterizar a amostra e suas relações com pais e professores. As variáveis estudadas na avaliação inicial (seis, oito e dez anos) e no seguimento de dois anos foram desempenho acadêmico e características pessoais-sociais (autoconceito, depressão, percepção em relação aos pais, ajustamento, comportamento disruptivo e relação com os pares). Seus resultados mostraram mais meninos que meninas com baixo desempenho. Mostraram, ainda, que quanto mais persistente é a trajetória de baixo desempenho escolar, mais a criança se torna disruptiva, impaciente, agressiva e menor é o apoio dos pais e professores. O menor apoio, por sua vez, provoca reflexos na educação, mais focalizada em disciplina e controle.

Sintetizando os resultados dos estudos revistos nesta seção, algumas convergências podem ser identificadas. A primeira tem relação com o modelo de trajetória de desenvolvimento proposto por Fergusson et al. (1993). Foi confirmada a proposição dos autores de que o transtorno de hiperatividade e atenção, por si só, prevê baixo desempenho escolar, mas não comportamento antisocial. É a comorbidade precoce da desatenção/hiperatividade com problemas de conduta que prediz comportamento anti-social (Fergusson et al., 1993; Fergusson et al., 1997; McGee et al., 2002; Rapport et al., 1999).

Outro ponto de convergência destes resultados é a forte validação da distinção entre comportamento hiperativo e antisocial. Ambos os conceitos oferecem, não só, informações importantes e diferentes, como predizem conseqüências adversas distintas. Apesar da associação entre eles, cada um está associado a um efeito adverso específico e descreve uma dimensão particular da psicopatologia na infância (Fergusson et al. 1997; McGee et al., 2000). De um lado, habilidades cognitivas e de atenção, e de outro, ajustamento social, irão predizer progressões desenvolvimentais muito distintas (Fergusson et al., 1993).

Alguns estudos revistos nesta seção retomaram a associação, já conhecida, entre adversidade ambiental e o desenvolvimento de problemas externalizantes. Nesse cenário, o estudo de McCall et al. (2000) faz sentido, ao demonstrar que a adversidade crônica, representada pela história persistente de fracasso escolar, aumenta as chances de a criança apresentar comportamentos agressivos e disruptivos na adolescência (não necessariamente transtornos), independente da presença inicial de problemas externalizantes.

Estudos sobre precursores da associação entre problemas de comportamento externalizantes e baixo desempenho escolar. 
Fazem parte desta categoria cinco artigos que se propõem a estudar os precursores da associação entre problemas de comportamento e baixo desempenho escolar, em amostras da comunidade, não identificadas com transtornos. Dois artigos selecionaram crianças com déficit de atenção e/ou hiperatividade (Rabiner \& Coie, 2000; Velting \& Grover, 1997) e três artigos selecionaram crianças com problemas de comportamento (Miles \& Stipek, 2006; Normandeau \& Guay, 1998; Sanson, Prior, \& Smart, 1996).

Quatro artigos iniciam a sua avaliação no jardim da infância (Miles \& Stipek, 2006; Normandeau \& Guay, 1998; Rabiner \& Coie, 2000; Velting \& Grover, 1997). Um estudo inicia nos 4-8 meses (Sanson et al., 1996).

Rabiner e Coie (2000) examinaram se existia uma relação preditiva entre problemas de atenção e desempenho em leitura. As variáveis avaliadas no jardim de infância foram desempenho em leitura (adaptado para a idade), atenção, inteligência, problemas internalizantes e externalizantes e envolvimento parental. Na primeira e na quinta série foi avaliado o desempenho em leitura. Estes autores enfatizaram a separação entre atenção e hiperatividade-impulsividade como variáveis distintas. Os resultados mostraram que problemas de atenção, mas não hiperatividade, prediziam desempenho em leitura, mesmo quando controladas as variáveis QI, desempenho de leitura anterior e envolvimento parental.

Velting e Grover (1997) investigaram a relação entre desatenção e hiperatividade em uma amostra de crianças de baixa renda. Os autores avaliaram comportamento e habilidades de pré-leitura no jardim de infância e comportamento e habilidades de leitura na primeira série. Os resultados mostraram uma forte relação entre desatenção-hiperatividade e habilidades de leitura, e entre habilidades de pré-leitura e habilidades de leitura. Os níveis de hiperatividade foram fortemente correlacionados no decorrer de três anos, no entanto, na pré-escola, desatençãohiperatividade não estava correlacionada com o desenvolvimento de habilidades de pré-leitura. Talvez isso se deva ao fato de que, no jardim da infância, as primeiras noções de leitura ainda não requerem altos níveis de atenção.

Miles e Stipek (2006) estudaram a relação entre habilidades sociais (comportamento pró-social versus agressividade) e desempenho em leitura no jardim de infância, primeira, terceira e quinta séries. Os resultados apontaram que baixo desempenho em leitura na primeira e na terceira séries predizia comportamento agressivo na terceira e na quinta séries, respectivamente. Mostraram também que crianças do jardim da infância e da primeira série com mais habilidades sociais tinham melhor desempenho em leitura, sugerindo que, em séries iniciais, habilidades sociais predizem bom desempenho em leitura. Porém essa associação diminuía na terceira e na quinta séries. É possível que crianças com habilidades pró-sociais venham a desenvolver hábitos de trabalho positivos, como ser atento, obediente e responsável para terminar suas tarefas. Já em séries avançadas, outros fatores como expectativas de sucesso e interesse em trabalho teriam mais importância para predizer formas de engajamento e aprendizado.

Normandeau e Guay (1998) investigaram se o autocontrole cognitivo na criança é mediador da relação entre comportamento na pré-escola e desempenho acadêmico na primeira série. Os autores analisaram, no jardim de infância, comportamento pró-social, agressividade, ansiedade-timidez e habilidades intelectuais; na primeira série, avaliaram desempenho acadêmico e autocontrole cognitivo. Os resultados revelaram que crianças menos agressivas e com mais comportamento pró-social na pré-escola exercem melhor controle sobre suas tarefas escolares na primeira série. $\mathrm{O}$ autocontrole cognitivo, por sua vez, está positivamente relacionado com desempenho acadêmico no fim da primeira série. Por outro lado, ansiedade-timidez não influencia o autocontrole cognitivo das tarefas escolares, tal como avaliado pelos autores. Assim, a hipótese de mediação é confirmada para agressividade, mas não para ansiedade-timidez, que neste estudo mostra apenas um efeito direto no desempenho escolar.

Sanson et al. (1996) compararam quatro grupos: $\mathrm{PC}^{2}+\mathrm{DL}$, $\mathrm{PC}$, DL e grupo controle, quanto ao comportamento, ao temperamento e à história familiar desde os quatro meses até os oito anos de idade. As variáveis relevantes analisadas foram: aos 4-8 meses, temperamento, percepção geral das mães sobre a criança, nível de escolaridade materna e ocupação paterna; com 1-3 anos, temperamento, hiperatividade e percepção geral da mãe sobre a criança; aos 3-4 anos, comportamento de hostilidadeagressividade, ansiedade-medo, hiperatividade, temperamento de inflexibilidade e persistência; aos 5-6 anos, comportamento de hostilidade-agressividade, ansiedade-medo, hiperatividade, temperamento de inflexibilidade e persistência, desempenho em leitura e coordenação motora. Os resultados mostraram que o grupo $\mathrm{PC}+\mathrm{DL}$ de meninos, em comparação com o grupo $\mathrm{PC}+\mathrm{DL}$ de meninas, era visto de forma mais negativa pela mãe (aos 4-8 meses); tinha mais problemas de comportamento e de temperamento (aos 4-8 meses e aos 1-3 anos); apresentava mais problemas de hiperatividade e agressividade-hostilidade (aos 3-4 e 5-6 anos). O grupo PC+DL também apresentou aos 5-6 anos, segundo o relato dos professores, mais indicadores de imaturidade (hiperatividade, hostilidade, agressividade, atraso em leitura, menor flexibilidade e orientação). Neste grupo se detectou ocupação paterna e educação materna baixas, o que sugere menos estimulação educacional e menos acesso a recursos.

Os resultados dos artigos revistos nesta seção parecem sugerir duas associações importantes. Em primeiro lugar, os artigos com amostras de crianças com problemas de atenção e hiperatividade encontraram forte relação entre desatençãohiperatividade e desempenho em leitura no seguimento. Em segundo lugar, os artigos com amostras de crianças com problemas de comportamento, ao associarem ao desempenho em leitura variáveis como temperamento e habilidades sociais (auto-confiança, cooperação, coordenação, socialização e autocontrole cognitivo), mostraram que comportamentos pró-sociais estavam diretamente associados a desempenho de leitura em séries iniciais.

Todos os artigos incluídos nesta seção avaliaram as crianças na pré-escola e na primeira série. A transição do jardim da infância para a primeira série é um momento crucial do desenvolvimento, porque há um acúmulo de demandas impostas ao indivíduo, como as primeiras noções de leitura, o desempenho 
acadêmico de forma geral e o ajustamento ao ambiente escolar. Estes estudos sugerem que a experiência da criança na escola com os seus pares, seu engajamento, atenção e comportamento podem mitigar ou exacerbar o risco de fracasso ou o potencial de sucesso.

Para finalizar esta discussão, cabe comentar alguns pontos. $\mathrm{O}$ primeiro diz respeito ao pequeno número de artigos identificados na literatura indexada, ou seja, dezoito, em 16 anos, relatando estudos longitudinais sobre a associação entre desempenho escolar e os comportamentos externalizantes nos anos do ensino fundamental. No entanto, este pequeno número é maior do que aquele identificado na revisão anterior, que cobriu igual período de tempo. Hinshaw (1992), usando critérios semelhantes aos desta pesquisa, encontrou 13 artigos no período de 1974 a 1990.

O segundo ponto a ser destacado é a convergência de resultados entre artigos. De forma geral, os resultados dos artigos parecem sugerir que: 1 . A associação $\mathrm{DL}+\mathrm{H}$ traz maior prejuízo psicossocial na adolescência que as condições DL e $\mathrm{H}$ isoladamente (Chadwick et al.,1999; Pisecco et al., 1996; Sanson et al., 1996; Smart et al., 1996); 2. hiperatividade e problemas de conduta estão associados a trajetórias distintas de desenvolvimento (Barkley et al., 1990; Fergusson et al., 1993; Fergusson et al., 1997; Fischer et al., 1993; McGee et al., 2002; Rapport et al., 1999); 3. Os problemas emocionais e de comportamento de adolescentes se manifestam predominantemente na escola (McCall et al., 2000; Pisecco et al., 1996; Smart et al., 1996); 4. TDAH prediz baixo desempenho em leitura (Biedermann et al., 1996; Latimer et al., 2003; Rabiner \& Coie, 2000; Rapport et al., 1999); 5. Baixo desempenho em leitura é mais comum em meninos (McCall et al., 2000) e a associação de dificuldades de leitura com problemas de comportamento também é maior neste gênero (Smart et al., 1996); 6. O impacto dos problemas de comportamento no desempenho escolar depende do momento de escolarização (Miles \& Stipek, 2006; Normandeau \& Guay, 1998; Rabiner \& Coie, 2000; Sanson et al., 1996; Velting \& Grover, 1997); 7. A criança com TDAH está mais exposta à instabilidade ambiental (Chadwick et al., 1999; Barkley et al., 1990; Biedermann et al., 1996; Sanson et al., 1996).

Outro aspecto a destacar é que alguns estudos apresentaram resultados controversos ao investigarem as causas da associação entre baixo desempenho escolar e problemas de comportamento. Enquanto McCall et al. (2000) encontraram indícios de que baixo desempenho leva a problemas de comportamento e Williams e McGee (1994) relataram que dificuldades de leitura aos nove anos levam a transtorno de conduta aos 15, Barkley et al. (1990), Smart et al. (1996), e Williams e McGee (1994) obtiveram resultados sugestivos de que problemas de comportamento levam a baixo desempenho em leitura ou pioram as dificuldades de leitura já existentes. O que esses resultados controversos parecem mostrar é a insuficiência do modelo explicativo para dar conta da complexidade do fenômeno. Há um reconhecimento crescente de que não se trata de identificar quais fatores levam a quais resultados. Em vez disso, o modelo causal linear vem sendo substituído por um modelo sistêmico multidimensional, transacional e evolutivo (desenvolvimental), que considera condições sediadas na criança e no ambiente, bem como as mutações nessas variáveis ao longo do tempo (acumulação, atenuação, supressão, aparecimento) e em momentos sensíveis (Masten et al., 2005).

Dada a natureza do problema em estudo, em um modelo com tais características o ambiente deveria estar representado por variáveis relevantes de pelo menos dois contextos - família e escola. No entanto, dentre os artigos revistos, aqueles que levaram em conta condições do ambiente se limitaram a variáveis do contexto familiar, excluindo fatores associados ao contexto escolar, com uma única exceção (McCall et al, 2000). Desse modo, fica limitado o alcance dos resultados, por não permitirem a discussão de aspectos relacionados à escola, como, por exemplo, as limitações do sistema escolar para lidar de forma competente com as diferenças individuais e desenvolver ao máximo as potencialidades de todos os alunos.

Especificamente em relação à lacuna identificada por Hinshaw (1992), relativa ao conhecimento disponível sobre os riscos psicossociais e os fatores de proteção associados à persistência e à remissão do problema, pode-se dizer que houve algum avanço nos modelos explicativos, passandose a considerar as variáveis ambientais não apenas como antecedentes, mas também como moderadores da associação entre comportamento externalizante e pobre desempenho escolar (Barkley et al., 1990; Biederman et al., 1996; Fischer et al., 1993; Rabiner \& Coie, 2000). No entanto, esse avanço foi modesto. Nas pesquisas que incluíram condições familiares, quando estas não foram tratadas como variáveis "confundidoras" (Fergusson et al., 1993; Fergusson et al., 1997; Rabiner \& Coie, 2000), apenas fatores de risco foram considerados.

No único estudo que incluiu em seu modelo de análise fatores de proteção em interação com fatores de risco, a estratégia se mostrou frutífera para identificar a influência atenuante das práticas parentais positivas sobre a manifestação dos problemas na adolescência (Latimer et al., 2003), um resultado que se alinha aos de um estudo recente com foco na transição para o ensino médio (Burchinal, Roberts, Zeisel, \& Rowley, 2008). É importante, então, registrar a necessidade de maior investimento de pesquisa na exploração das condições de proteção do ambiente, não só para melhor conhecimento dos processos envolvidos no curso dos problemas em foco, mas, também, pela potencial contribuição para programas de prevenção.

Mesmo com essas limitações, observa-se, no período coberto pela revisão, um progresso em relação à revisão feita por Hinshaw (1992) com a adoção, em alguns artigos, de modelos multifatoriais de trajetórias de desenvolvimento. Como decorrência da maior sofisticação metodológica, a evidência acumulada hoje valida claramente a distinção entre comportamento hiperativo e anti-social, elucidando uma importante questão levantada por Hinshaw na conclusão de seu estudo.

Pesquisas longitudinais são de grande valor para reconhecer fatores de risco e de proteção, que parecem ser a chave para a identificação precoce de crianças vulneráveis e com necessidade de tratamento especializado em serviços de saúde mental. $\mathrm{O}$ adequado manejo, encaminhamento e tratamento do caso dependem de uma identificação ativa das condições de risco e dos recursos protetores que podem ser mobilizados. Nesse contexto, a 
queixa escolar adquire um significado mais amplo. É possível que essas famílias tenham expectativas de receber apoio para lidar também com outros problemas, provavelmente intensificados com a dificuldade acadêmica (Marturano \& Elias, 2006).

Entender a magnitude do problema da associação entre baixo desempenho escolar e comportamentos externalizantes e suas conseqüências adversas tem implicações diretas para os cuidados de saúde mental da população infantil com queixa escolar. Tal entendimento pode contribuir para o planejamento de práticas mais assertivas de tratamento e de prevenção, implementadas por meio de treinamento e preparo de profissionais de saúde mental, e para uma melhor organização dos serviços.

\section{Referências}

Achenbach, T. M., \& Edelbrock, C. (1978). The classification of child psychopathology: a review and analysis of empirical efforts. Psychological Bulletin, 85, 1275-1301.

Arnold, D. H. (1997). Co-occurrence of externalizing behavior problems and emergent academic difficulties in high-risk boys: a preliminary evaluation of patterns and mechanisms. Applied Developmental Psychology, 18, 317-330.

Barbosa, J. I., \& Silvares, E. F. (1994). Uma caracterização preliminar das clínicasescola de Fortaleza. Estudos de Psicologia, 11, 50-56.

Barkley, R. A., Fischer, M., Edelbrock, C., \& Smallish, L. (1990). The adolescent outcome of hyperactive children diagnosed by research criteria: I. An 8-year prospective follow-up study. Journal of American Academy Child Adolescent Psychiatry, 29, 546-557.

*Biederman, J., Faraone, S., Milberger, S., Guite, J., Mick, E., Chen, L., ... Perrin, J. (1996). A prospective 4-year follow-up study of attention-deficit hyperactivity and related disorders. Archives of General Psychiatry, 53, 437-446.

Burchinal, M. R., Roberts, J. E., Zeisel, S. A., \& Rowley, S. J. (2008). Social risk and protective factors for African American children's academic achievement and adjustment during the transition to middle school. Developmental Psychology, 44, 286-292.

*Chadwick, O., Taylor, E., Taylor, A., Heptinstall, E., \& Danckaerts, M. (1999). Hyperactivity and reading disability: a longitudinal study of the nature of the association. Journal of Child Psychology and Psychiatry, 40, 1039-1050.

Cury, C. R., \& Golfeto, J. H. (2003). Strengths and Difficulties Questionnaire (SDQ): a study of school children in Ribeirão Preto. Revista Brasileira de Psiquiatria, 25, 139-145.

Dodge, K. A., \& Pettit, G. S. (2003). A biopsychosocial model of the development of chronic conduct problems in adolescence. Developmental Psychology, 39, 349-371.

Ellis, A., \& Bernard, M. E. (2006). Rational emotive behavioral approaches to childhood disorders: theory, practice and research. New York: Springer Science.

*Fergusson, D. M., Horwood, L. J., \& Lynskey, M. T. (1993). The effects of conduct disorder and attention deficit in middle childhood on offending and scholastic ability at age 13. Journal of Child Psychology and Psychiatry, $34,899-916$

*Fergusson, D. M., Lynskey, M. T., \& Horwood, L. J. (1997). Attention difficulties in middle childhood and psychosocial outcomes in young adulthood. Journal of Child Psychology and Psychiatry, 38, 633-644.
*Fischer, M., Barkley, R. A., Fletcher, K. E., \& Smallish, L. (1993). The adolescent outcome of hyperactive children: predictors of psychiatric, academic, social, and emotional adjustment. Journal of the American Academy of Child and Adolescent Psychiatry, 32, 324-332.

Hinshaw, S. P. (1992). Externalizing behavior problems and academic underachievement in childhood and adolescence: causal relationships and underlying mechanisms. Psychological Bulletin, 111, 127-155.

*Latimer, W. W., August, G. J., Newcomb, M. D., Realmuto, G. M., Hektner, J. M. \& Mathy, R. M. (2003). Child and familial pathways to academic achievement and behavioral adjustment: a prospective six-year study of children with and without ADHD. Journal of Attention Disorders, 7, 101-116.

Marturano E. M., \& Elias, L. C. S. (2006). Atendimento psicológico a crianças com dificuldades escolares. In E. F. M. Silvares (Org.), Atendimento psicológico em clínicas-escola (pp.75-90). Campinas, SP: Alínea.

Marturano E. M., Toller G. P., \& Elias, L. C. S. (2005). Gênero, adversidade e problemas sócio-emocionais associados à queixa escolar. Estudos de Psicologia, 22, 371-380.

Masten, A. S., Roisman, G. I., Long, J. D., Burt, K. B., Obradovic, J., Riley, J. R., ...Tellegen, A. (2005). Developmental cascades: linking academic achievement and externalizing and internalizing symptoms over 20 years. Developmental Psychology, 41, 733-746.

*McCall, R. B., Scott, R. B., \& Lau, S. (2000). The nature and correlates of underachievement among elementary schoolchildren in Hong Kong. Child Development, $71,785-801$.

*McGee, R., Prior, M., Williams, S., Smart, D., \& Sanson, A. (2002). The long-term significance of teacher-rated hyperactivity and reading ability in childhood: findings from two longitudinal studies. Journal of Child Psychology and Psychiatry, 43, 1004-1017.

*Miles, S. B., \& Stipek, D. (2006). Contemporaneous and longitudinal associations between social behavior and literacy achievement in a sample of low-income elementary school children. Child Development, 77, 103-117.

*Normandeau, S., \& Guay, F. (1998). Preschool behavior and first-grade school achievement: the mediational role of cognitive self-control. Journal of Educational Psychology, 90, 111-121.

*Pisecco, S., Baker, D. B., Silva, P. A., \& Brooke, M. (1996). Behavioral distinctions in children with reading disabilities and/or ADHD. Journal of American Academy Child Adolescent Psychiatry, 35, 1477-1484.

*Rabiner, D., \& Coie, J. D. (2000). Early attention problems and children's reading achievement: a longitudinal investigation. Journal of the American Academy of Child and Adolescent Psychiatry, 39, 859-867.

*Rapport, M. D., Scanlan, S. W., \& Denney, C. B. (1999). Attention-deficit/ hyperactivity disorder and scholastic achievement: a model of dual development pathways. Journal of Child Psychology and Psychiatry, 40, 1169-1183.

*Sanson, A., Prior, M., \& Smart, D. (1996). Reading disabilities with and without behavior problems at 7-8 years: Prediction from longitudinal data from infancy to 6 years. Journal of Child Psychology and Psychiatry, 37, 529-541.

*Smart, D., Sanson, A., Prior, M. (1996). Connections between reading disability and behavior problems: testing temporal and causal hypotheses. Journal of Abnormal Psychology, 24, 363-383.

*Velting, O. N., \& Grover, J. W. (1997). Inattention-hyperactivity and reading achievement in children from low-income families: a longitudinal model. Journal of Abnormal Child Psychology, 25, 321-331.

*Williams, S., \& McGee, R. (1994). Reading attainment and juvenile delinquency. Journal of Child Psychology and Psychiatry, 35, 441-459. 
Lylla Cysne Frota D'Abreu, mestre em Ciências Médicas pela Faculdade de Medicina de Ribeirão Preto da USP, é doutoranda pela Universidade de Potsdam, Alemanha. E-mail: lyllacysne@yahoo.com.br Edna Maria Marturano, doutora em Ciências (Psicologia) pela Universidade de São Paulo, é professora titular e livre-docente em Psicologia pela Faculdade de Medicina de Ribeirão Preto da USP. Endereço para correspondência: Rua Tenente Catão Roxo, 2650, 14051-140, Ribeirão Preto, SP, Brasil. E-mailemmartur@fmrp.usp.br 